\title{
Engendering Empowerment:
}

Education and Equality

Dakar, Senegal, 17-20 May 2010

\section{Contested development? Women's economic empowerment and intimate partner violence in urban and rural Tanzania}

Authors: Seema Vyas MA; Jessie Mbwambo MD; Charlotte Watts PhD.

\section{Introduction}

Globally, one of the most common forms of violence against women is that perpetrated by a husband or other intimate male partner (Krug 2002). Intimate partner violence (IPV) takes a variety of forms, including physical violence (ranging from slaps, punches and kicks to assaults with a weapon and homicide) and sexual violence (including forced or coerced sex, or forced participation in degrading sexual acts). A population based study found that between $15 \%$ and $71 \%$ of women have ever been physically and or sexually assaulted by their partners at some point in their lives (Garcia-Moreno et al. 2006).

Physical and sexual IPV against women has been shown to adversely affect women's health, to limit the degree to which women are able to work, earn an income, or independently make decisions about their health and their children's schooling and use of health services, and therefore, it is an important barrier to development.

Increasingly, studies are focussing attention on understanding the social determinants of IPV. A recent systematic review of published studies found evidence related to the strength of association between different indicators of women's and men's empowerment and women's ever and past year risk of IPV in low and middle income countries (Vyas and Watts 2009). The review found that household poverty or low socioeconomic status (SES) was associated with women's risk of violence; that women's secondary education, and to some extent men's secondary education, was generally protective but that there was a less strong evidence of a protective effect of primary education. The reasons for this may be that secondary education or higher education give women greater options not to marry a man who they think are violent or to leave violent relationships, or it increases women's communication skills enabling them to better deal with spousal conflict. It may also be that 
women with higher education are more valued by their partner. However, there was also some evidence that women were at increased risk of IPV when they had a higher educational attainment than their partner. The findings on women's employment and risk of IPV was mixed, for example, out of 11 studies included in the review that assessed this link, five found a protective association and six documented a risk association with past year experience of IPV.

The WHO multi-country study on women's health and domestic violence documented prevalence of physical and or sexual IPV between $31 \%$ and $49 \%$ in the three SSA countries participating in the study (Garcia-Moreno 2006). Despite the pervasiveness of violence, there has been extremely limited analysis from the region about the extent to which women's empowerment may reduce or exacerbate women's risk of violence. The purpose of this study is to bridge this gap in evidence using data collected from Tanzania as part of the WHO multi-country study.

\section{Tanzania setting}

The population of Tanzania is approximately 40 million and with a GNI per capita of $\$ 410$ it is one of the poorest in the world. In Tanzania it is recognised that women play an important role in contributing to the country's domestic economy. However, as well as widespread norms condoning violence and promoting traditional gender roles, there is substantial inequality between the sexes - with some of the lowest gender empowerment scores in the world (UNDP 2005). Findings from the 2004 - 2005 DHS documented that women in Tanzania have fewer educational and economic opportunities than men, are less likely to pursue higher education and are more disproportionately affected by poverty (DHS 20042005).

\section{Theoretical approach}

The analysis used in this study draws upon the sociological theories that have linked economic resources (hereafter termed economic status or economic empowerment and both are used interchangeably) with women's risk of IPV. One of the earlier theories, resource theory, posits higher IPV in households characterised by lower SES and as men are traditionally the head of households with men who have lower economic status (e.g. 
education attainment or income) (Goode 1971). The higher violence associated with lower SES is mediated through either the increased stress of poverty or because men compensate for their lack of economic status.

This has been expanded to more explicitly to consider the relative differentials in economic status (Relative resource theory) (McCloskey 1996; Macmillan and Gartner, 1999). According to Macmillan and Gartner (1999) economic status has symbolic gender identities and for men is an important factor linked with constructing masculinity. Therefore, relative resource theory examines the economic status of both partners on its influence on violence, suggesting that women who have more education than their partner, or who are employed when their partner is not, or who have a higher income than their partner are at a higher risk of violence.

While resource theory focuses on violence and its association with the man's economic status, marital dependency theory argues that it is factors associated with the individual woman, rather than her partner, that influences her risk of violence. Thus women with less economic status are at increased risk of IPV (Kalmuss and Straus 1982; Dobash and Dobash 1979). IPV is thus mediated through women's higher tolerance of violence as women with less education or women who are unemployed are trapped in their marriage; are less able to negotiate behaviour change in their partner; and have fewer alternatives to marriage and less likely to leave or seek intervention (Kalmuss and Straus 1982; Gelles 1976; Strube and Barbour 1983).

Finally, the ecological model put forward by Heise (1998) recognises that the absolute or relative levels of education or employment that women and men have within a partnership are potentially influential, but the role of other contextual factors is also more explicitly acknowledged. These contextual factors could include early life experience of violence such as childhood abuse or witnessing violence in childhood; or situational factors e.g. whether she has a child or other dependents that the perpetrator is supporting, the age of her children, or her partner's use of alcohol and drugs. 
Guided by these theoretical models, that present competing predictions about the link between economic empowerment and IPV, this analysis aims to understand the relationship between different indicators of women's and men's economic empowerment and women's risk of lifetime and current partner violence in two contrasting Tanzania settings.

\section{Methods}

\section{Setting and data}

The data used for this study comes from the WHO multi-country study on women's health and domestic violence. ${ }^{1}$ The study in Tanzania was a cross-sectional household survey of ever partnered women aged between 15-49 in two sites, Dar Es Salaam (DES) Tanzania's largest city; and Mbeya, a provincial region. Data were collected between November 2001 and March 2002 and the survey used a multi-stage, cluster design. ${ }^{2}$ The overall individual response rate was very high with $96 \%$ of eligible women agreeing to participate.

\section{Questionnaire}

The questionnaire used for this study was developed by the WHO and was used in all the multi-country studies, with some setting specific adaptations for the Tanzanian context. Female interviewers administered the survey face to face in the local language (Kiswahili) and in private.

\section{Measure of intimate partner violence (IPV)}

The survey recorded responses of six different acts of physical violence and three different acts of sexual violence by an intimate partner. The acts of physical violence ranged from moderate acts slapped or having something thrown; pushed or shoved; hit with fist; and kicked or dragged, to more severe acts choked or burned; threatened with a weapon. A further three questions were asked on whether the woman experienced forced sex; was afraid to say no to sex; or forced to do a degrading / humiliating act. Data were collected for lifetime / ever and for current (12 month prior to interview) experience. Prevalence of

\footnotetext{
${ }^{1}$ The multi-country study was conducted in 15 sites from 10 countries. Dr. Mbwambo was the Tanzania PI and Professor Watts was one of the core research teams.

${ }^{2}$ Details of sample characteristics in Mbwambo, Vyas et al. In preparation
} 
physical and or sexual violence was identified if a woman reported yes to any of the six acts of physical violence or any of the three acts of sexual violence.

\section{Measures of economic empowerment}

Measures of economic empowerment were based on 1) household SES; 2) Education attainment; 3) Employment status; and 4) Contribution to household income. Household SES was measured by creating an asset based index using the data collected on household ownership of durable assets and housing infrastructure characteristics (Vyas \& Kumaranayake 2006). Education attainment was classified into four categories: No schooling; incomplete primary schooling; complete primary schooling; and some secondary or higher. Education attainment of the partner was categorised in the same way. Relative education attainment was measured with four categories: both the respondent and her partner had no schooling; the partner has a higher education attainment; the respondent has a higher education attainment; and both have the same level of education. The employment status of the respondent and her partner was also examined and relative employment status was categorised into: neither working; partner only working; respondent only working; and both working. Relative contribution to household income was categorised as: neither working; partner contributes all or more to the household income; respondent contributes all or more to the household income; and both contribute the same. 


\section{Other covariates}

Other covariates considered in the analysis included women's and their partners sociodemographic and behavioural characteristics. Women's socio-demographic characteristics included her age; current partnership status; religion; and whether she has a child. Women's behavioural characteristic was measured by her alcohol use (never or less than once a week; at least once a week) and her childhood characteristics were whether she grew up in an urban or a rural location; whether or not her mother was beaten by her father; mean age of $1^{\text {st }}$ sex; and whether she experienced non partner physical sexual abuse. Characteristics of women's partners used in this analysis include their socio-demographics: age; and whether he was a polygamist. Behavioural characteristics included whether he has other women; and whether his alcohol use was problematic i.e. causing money; family or any other problems.

\section{Analysis}

Data were analysed using SPSS v 16.0 and the sample used for analysis were ever partnered women who answered questions on their experience of IPV (1442 DES; 1256 Mbeya). Multivariate logistic regression was used to explore the association between lifetime and current IPV and women's and their partners characteristics.

\section{Ethics}

The study adhered to ethical guidelines on researching violence against women, and ethical clearance was sought from WHO's ethical review group and from the local Tanzanian ethics board at $\mathrm{MUCHS}^{3}$ (WHO). Interviewers sought informed consent verbally with individual respondents at the start of each interview. A list of local women's related organizations was given to all interviewed women who agreed to take it.

\footnotetext{
${ }^{3}$ Muhimibili University College of Health Sciences now Muhimbili University of Health and Allied Sciences (MUHAS)
} 


\section{Results}

Table 1 shows the prevalence of lifetime and current IPV among ever partnered women in the two study sites. Forty-one percent of women in DES reported they had experienced physical and or sexual violence by an intimate partner in their lifetime and 22 percent reported experience in the 12 months to interview. Lifetime and current experience of partner violence was higher in the provincial region, Mbeya, with prevalence of 56 percent and 29 percent respectively. Table 1 also shows the socio-demographic characteristics of women experiencing lifetime and current IPV and compares them with the total sample of women.

Compared to total site sample, respondents in DES who had completed primary school education were more likely to report lifetime and current IPV, however, respondents at the lowest and the highest end of the education attainment category (i.e. with no schooling or with some secondary or more schooling) were less likely to report lifetime or current IPV. Ever abused women were also more likely to be engaged in income generating activities, but there was no difference with current IPV.

In Mbeya, respondents with no schooling or with incomplete primary schooling were more likely to report lifetime IPV, and respondents with complete primary education were less likely to report lifetime IPV. However, these associations were not seen when assessing the relationship with current IPV.

In both sites, ever or currently abused women were more likely to be in a cohabiting (living with a man but not married) relationship and to drink alcohol. They were also more likely to have had their first sexual encounter at a younger age, have experienced non-partner sexual abuse, and to report that their mother had been hit by their father. In both sites, there was no difference, when comparing abused women with the total site sample, by religion, childhood residence or experience of non-partner physical abuse (which was most commonly perpetrated by a teacher). 
Table 2 shows the socio-demographic characteristics of the women's partners. In DES, respondents whose partners had completed primary education were more likely to report lifetime or current abuse. In Mbeya, women who reported their partner had low education levels (no education or some primary education) were more likely to experience ever abuse and this relationship held for women whose partner had no schooling and current abuse. Some secondary or more education was protective in both sites and with both ever and past year experience of IPV. There was no difference between partners employment and ever and current abuse in either site. Household SES was not associated with violence in DES, however, the mean crowding index was higher for ever abused and currently abused women. Respondents in Mbeya categorised as low SES were more likely to report ever abuse and respondents in medium and high SES were less likely to report ever abuse.

In DES, there was no difference in the partners mean age for ever abused women and the sample as a whole. However, perpetrators of current abuse were more likely to be younger than the sample average. In Mbeya, the average age of women's partners was higher for ever abused women and lower for currently abused women. Women whose partner was polygamous were more likely to experience ever abuse, and this relationship held with current abuse in Mbeya. In DES and Mbeya ever or currently abused women were more likely to report that their partner had other women and also that their partner's alcohol use was problematic.

The results of the logistic regression analysis (controlling for respondents age) are shown in Table 3. The analyses revealed a significant positive relationship between household crowding and lifetime or current IPV in DES and with lifetime IPV in Mbeya. However, the relationship with household SES was less clear. There was no significant association between SES and either lifetime or current IPV in DES, but respondents in low SES households were 2 times more likely to report lifetime IPV though this association was not significant with current IPV.

There appears to be a curvi-linear relationship between women's education and both lifetime and current IPV in DES as women with no formal schooling were less, but not significantly, likely to report IPV compared to women with some secondary or higher education. However, women with some primary were 1.6 times more likely to report lifetime IPV and 1.8 times more likely to report current IPV. In Mbeya, respondents with no 
schooling or with some primary schooling were significantly more likely to report lifetime IPV, however, this relationship was not significant with current IPV. In both sites, respondents whose partner had some secondary or more schooling had a significantly lower odds of experiencing lifetime or current IPV. However, a decreasing educational gradient was only observed in Mbeya. In DES respondents with a higher level of education than their partner had a higher, but not significant odds, or reporting lifetime and current IPV compared with respondents who had the same level of education as their partner. In Mbeya, respondents who were more educated than their partners were 1.5 times more likely to report lifetime abuse and 1.7 times more likely to report current abuse when compared to the reference category. In addition, respondents in partnerships where both had no formal schooling were also significantly more likely to report current IPV.

While respondents who were engaged in income generating activities were significantly more likely to report lifetime IPV in DES, this finding is hard to interpret as the temporal nature of both the IPV and whether the woman was employed at the time could not be established and the association was not significant with current IPV. Relationships where the partner only worked reduced the respondents odds of lifetime IPV in DES, however, women who contribute all or more to the household income were significantly more likely to experience current abuse in DES. There was no significant association between either the woman's or her partner's employment status or contribution to the household income and lifetime or current IPV in Mbeya.

In the multivariate analysis (controlling for contextual factors shown in Table 1 and Table 2), the risk association with household crowding was the only economic related factor that remained significant. In Mbeya, the protective association between higher partner education attainment and IPV remained significant and so did the risk association when a woman has more education than her partner. In both sites, factors that were associated with IPV were the respondents report that their mother had been hit by their father, low mean age of $1^{\text {st }}$ sex; their partners drinking and problematic alcohol use and whether he has had relationships with other women. Women in cohabiting and dating relationships and who had experienced non partner sexual abuse had a higher risk association of IPV in DES. In Mbeya the partner's involvement in fights with other men was significantly associated with higher IPV. 


\section{Discussion}

The findings from this study reveal that intimate partner violence against women in Tanzania is prevalent. Over 40 percent of respondents in DES and 56 percent of respondents in Mbeya reported that they had experienced physical and or sexual violence by an intimate partner at one point in their lifetime. Therefore, a greater understanding of the factors that are associated with IPV is essential.

This analysis provides some evidence on how the different indicators of men and women's economic empowerment are associated with IPV. However, it is important to note that due to the cross sectional nature of the survey it is difficult to establish the causal process i.e. does the stress of poverty cause violence in the household or does violence keep households poor. A further limitation is that stigma and fear could have prevented some respondents from disclosing IPV or that there may be lower reporting among women who find wife beating acceptable, and so weakening this studies ability to identify significantly the factors associated with violence.

The evidence from this study revealed that poverty reduction, male and female access to secondary education and reductions in inequality in education may have important protective impacts on the levels of IPV. There was, however, limited evidence of an association between women's employment and IPV and the results do not support the theory that women's access to income leads to an improvement in women's situation within the household. There was a strong inverse association between household SES and women's education attainment and acceptance of wife beating, with women who had some secondary or more schooling (compared to women in the other three education categories) being significantly less likely to accept that it is justifiable for a man to beat his wife. Women who were employed were also less likely to accept that it is justifiable for a man to beat his wife (Table 4). 


\section{References}

Ahmed, S., M. Koenig, and R. Stephenson, Effects of domestic violence on perinatal and early-childhood mortality: evidence from north India. Am J Public Health, 2006. 96(8): p. 1423-1428.

Campbell, J., Health consequences of intimate partner violence. Lancet, 2002. 359: p. 13311336.

Diop-Sidibe, N., Campbell, J.C., Becker, S. Domestic violence against women in Egypt - wife beating and health outcomes. Social Science and Medicine, 2006

Dobash, R. and R. Dobash, Violence against wives: A case against patriarchy. 1979, New York: Free Press.

Dunkle, K.L., et al., Perpetration of partner violence and HIV risk behaviour among young men in the rural Eastern Cape, South Africa. Aids, 2006. 20(16): p. 2107-14.

Garcia-Moreno, C., et al., Prevalence of intimate partner violence: findings from the WHO multi-country study on women's health and domestic violence. Lancet, 2006. 368(9543): p. 1260-9.

Gelles, R.J., Abused wives: why do they stay. Journal of Marriage and the Family, 1976. 38(4): p. 659-668.

Gibson-Davis, C., et al., Employment and the risk of domestic abuse among low-income women. Journal of Marriage and Family, 2005. 67: p. 1149-1168.

Goode, W. Force and violence in the family. Journal of Marriage and the Family, 1971. 33: $p$. 624-636.

Heise, L., Violence against women: An integrated, ecological framework. Violence Against Women, 1998. 4: p. 262-290.

Jejeebhoy, S.J., Associations between wife-beating and fetal and infant death: impressions from a survey in rural India. Studies in Family Planning, 1998. 29(3): p. 300-8.

Kalmuss, D.S. and M.A. Straus, Wife's marital dependency and wife abuse. Journal of Marriage and the Family, 1982. 44(2): p. 277-286.

Krug, E., et al., World report on violence and health. World Health Organization, Geneva, 2002.

Macmillan, R. and R. Gartner, When she brings home the bacon: Labour-force participation and the risk of spousal violence against women. Journal of Marriage and the Family, 1999. 61(4): p. 947-958. 
Mbwambo, J. Vyas, S. and C. Watts, The prevalence and correlates of intimate partner violence in Tanzania: findings from the WHO multi-country study. Draft paper, to be submitted to the WHO Bulletin.

McCloskey, L.A., Socioeconomic and coercive power within the family. Gender and Society, 1996. 10(4): p. 449-463.

Strube, M.J. and L. Barbour, The decision to leave an abusive relationship: economic dependence and psychological commitment. Journal of Marriage and the Family, 1983. 45(4): p. 785-793.

UNDP, Human Development Report 2005. 2005.

Vyas, S. and L. Kumaranayake, How to do (or not to do)... Constructing socio-economic status indices: How to use principal components analysis. Health Policy and Planning, 2006. 21(6): p. 459-468.

Vyas, S and C. Watts, How does economic empowerment affect women's risk of intimate partner violence in low and middle income country settings?: a systematic review of published evidence. Journal of International Development. 2009 21(5), 577-602. 


\section{Mbeya}

\begin{tabular}{|c|c|c|c|c|c|c|c|c|c|c|c|}
\hline \multirow[b]{3}{*}{ Total } & \multicolumn{5}{|c|}{ Dar Es Salaam } & \multicolumn{6}{|c|}{ Mbeya } \\
\hline & \multicolumn{2}{|c|}{ Sample \% (n) } & \multicolumn{2}{|c|}{ Lifetime IPV \% (n) } & Current IPV \% (n) & \multicolumn{2}{|c|}{ Sample \% (n) } & \multicolumn{2}{|c|}{ Lifetime IPV \% (n) } & \multicolumn{2}{|c|}{ Current IPV \% (n) } \\
\hline & 100.0 & $(1442)$ & 41.3 & $(596)$ & $21.5 \quad(313)$ & 100.0 & $(1256)$ & 55.9 & $(702)$ & 29.1 & (365) \\
\hline Respondent mean age (SD) & 30.45 & $(8.53)$ & 30.63 & $(8.19)$ & $27.81 \quad(7.19)$ & 29.71 & $(8.00)$ & 30.34 & $(7.87)$ & 28.16 & $(7.36)$ \\
\hline \multicolumn{12}{|l|}{ Current Partnership Status } \\
\hline Currently married & 57.4 & (828) & 51.0 & (304) & $51.4 \quad(161)$ & 55.2 & (693) & 52.6 & (369) & 55.1 & (201) \\
\hline Living with partner not married & 17.5 & (252) & 22.7 & (135) & $25.2(79)$ & 27.8 & (349) & 30.9 & (217) & 36.4 & (133) \\
\hline With partner not living together & 17.4 & (251) & 16.6 & (99) & $18.8 \quad(59)$ & 5.9 & (74) & 4.6 & (32) & 4.9 & (18) \\
\hline \multicolumn{12}{|l|}{ Religion } \\
\hline None & 0.0 & (0) & 0.0 & (0) & $0.0(0)$ & 10.8 & (136) & 12.8 & (90) & 12.6 & (46) \\
\hline Islam & 61.2 & (883) & 58.9 & (351) & $58.8 \quad(184)$ & 3.7 & (47) & 2.7 & (19) & 3.3 & (12) \\
\hline Christian & 37.5 & (541) & 40.4 & (241) & 40.6 (127) & 75.7 & (951) & 76.2 & (535) & 75.9 & (277) \\
\hline Other & 1.2 & (18) & 0.7 & (4) & $0.6 \quad(2)$ & 9.7 & (122) & 8.3 & (58) & 8.2 & (30) \\
\hline \multicolumn{12}{|l|}{ Respondent education } \\
\hline None & 13.2 & (190) & 10.7 & (64) & $8.6 \quad(27)$ & 24.4 & (306) & 27.6 & (194) & 25.8 & (94) \\
\hline Some secondary or higher & 22.9 & (330) & 19.6 & (117) & $19.5 \quad(61)$ & 7.8 & (98) & 7.0 & (49) & 7.1 & (26) \\
\hline Respondent earns money & 48.9 & (705) & 52.9 & (315) & $48.6 \quad(152)$ & 65.1 & (818) & 66.2 & (465) & 62.7 & (229) \\
\hline \multicolumn{12}{|l|}{ Child under 5} \\
\hline No children & 19.7 & (284) & 15.4 & (92) & $18.5 \quad(58)$ & 10.8 & (136) & 9.0 & (63) & 10.2 & (37) \\
\hline Child less than 5 & 45.8 & (661) & 47.5 & (283) & 51.4 (161) & 64.2 & (806) & 63.8 & (447) & 71.7 & (261) \\
\hline Children over 5 only & 34.5 & (497) & 37.1 & (221) & $30.0 \quad(94)$ & 24.9 & (313) & 27.2 & (191) & 18.1 & $(66)$ \\
\hline \multicolumn{12}{|l|}{ Drinks alcohol } \\
\hline Never / Rarely (less than once month) & 90.2 & (1298) & 86.9 & (518) & $86.3 \quad(270)$ & 73.5 & (923) & 67.8 & (475) & 66.2 & (241) \\
\hline More than once a month & 9.8 & (141) & 13.1 & (78) & $13.7 \quad(43)$ & 26.5 & (332) & 32.2 & (226) & 33.8 & (123) \\
\hline \multicolumn{12}{|l|}{ Childhood residence } \\
\hline Urban childhood & 54.9 & (791) & 55.5 & (331) & $59.1 \quad(185)$ & 13.5 & (170) & 13.2 & (93) & 12.6 & (46) \\
\hline Rural childhood & 45.1 & (651) & 44.5 & (265) & $40.9 \quad(128)$ & 86.5 & (1086) & 86.8 & (609) & 87.4 & (319) \\
\hline Respondent mean age 1st sex (SD) & 18.01 & $(3.37)$ & 17.55 & $(2.48)$ & $17.42 \quad(2.32)$ & 17.46 & $(2.35)$ & 17.16 & $(2.12)$ & 17.16 & $(2.09)$ \\
\hline Non partner physical abuse after 15 & 17.5 & (253) & 19.5 & (116) & $18.8 \quad(59)$ & 13.7 & (172) & 15.1 & (106) & 16.2 & (59) \\
\hline
\end{tabular}


E4 Conference

www.e4conference.org

Non partner sexual abuse (any age)

12.1 (175)

$17.6 \quad(105)$

$18.5 \quad(58)$

11.5 (144)

$13.5 \quad(95)$

$15.3 \quad(56)$

\section{Mother abused by father}

No

Yes

$51.8 \quad(746)$

$29.5(425)$

$43.5 \quad(259)$

18.7 (270)

36.7 (219)

$43.1 \quad(135)$

19.8 (118)

$40.6 \quad(127)$

40.1 (504)

35.2 (247)

$47.4(595)$

52.1 (366)

32.1 (117)

16.3 (51)

12.5 (157)

12.7 (89)

$55.3 \quad(202)$

$12.6 \quad(46)$ 
Mbeya

Sample \% (n) Lifetime IPV \% (n)

Current IPV \% (n)

Sample \% (n) Lifetime IPV \% (n) 38.27 (10.41)

Current IPV \% (n)

36.89 (10.71)

37.68 (10.83)

35.57 (10.84)

\section{Relationship type}

Monogomous

Polygamous

79.9 (1014)

$14.0 \quad(178)$

$75.8 \quad(420)$
17.1

17.1 (95)

Partner has other women

Yes

May have / Don't know

Mean household crowding index (SD)

$19.8(285)$

51.7 (742)

$28.5(409)$

$29.3 \quad(174)$

$44.8 \quad(266)$

25.9 (154)

$2.57(1.16)$

$2.65 \quad(1.23)$

Household SES

High

Medium

Low

\section{Partner education}

None

Some primary

Complete primary

Some secondary +

Attended school but don't know level

\section{Partner employed}

\section{Problems associated with alcoho}

Relative education

Both have none

Partner has more

Woman has more

Both have same

Relative employment status

Neither employed

Partner only employed

Woman only employed

Both employed $\begin{array}{ll}12.0 & (173) \\ 23.4 & (338)\end{array}$

$\begin{array}{ll}23.4 & (338) \\ 64.6 & (931)\end{array}$

$10.9 \quad(65)$

$22.5 \quad(134)$

66.6 (397)

$10.9 \quad(34)$

21.1 (66)

68.1 (213)

\section{$5.3(76)$}

$6.8(98)$

$45.4(654)$

39.2 (564)

3.3 (47)

$$
\begin{aligned}
4.9 & (29) \\
6.7 & (40) \\
51.0 & (304) \\
14.2 & (204) \\
3.2 & (19)
\end{aligned}
$$

$4.5 \quad$ (14)

5.1 (16)

52.1 (163)

34.8 (109)

3.5 (11)

87.1 (1252)

$88.2 \quad(525)$

$88.5 \quad(277)$

6.5 (94)

$11.1 \quad(66)$

\begin{abstract}
1.9 (27)
$37.0 \quad$ (519)

11.3 (159)

$49.8 \quad(698)$
\end{abstract}

$\begin{array}{cc}1.0 & (6) \\ 35.9 & (208) \\ 13.4 & (78) \\ 49.7 & (288)\end{array}$

$49.7 \quad(288)$

6.6 (95)

$44.5 \quad(640)$

$6.3 \quad(90)$

$42.6 \quad$ (612)
$5.5 \quad$ (33)

$41.7 \quad(248)$

$6.2(37.0)$

$46.6 \quad$ (277)
1.3 (4)

$35.6 \quad(108)$

$12.5 \quad$ (38)

6.1 (19)

45.4 (142)

5.4 (17)

43.1 (135)
76.2 (214)

$14.2(40)$

9.6 (27)

$29.6 \quad(92)$

$42.4 \quad$ (132)

$28.0 \quad(87)$

$2.74 \quad(1.28)$

\begin{abstract}
75.2 (905)
23.4 (281)
\end{abstract}

1.4 (17)

$\begin{aligned} 70.0 & (480) \\ 28.6 & (196) \\ 1.5 & (10)\end{aligned}$

$70.8 \quad(250)$
27.5

27.5 (97)

1.7 (6)

22.9 (288)

56.7 (711)

20.4 (256)

$29.9 \quad(210)$

$46.0 \quad$ (323)

24.1 (169)

$32.6 \quad(119)$

$46.0 \quad$ (168)

21.4 (78)

$2.64(1.07)$

$2.69 \quad(1.09)$

$2.73 \quad(0.98)$

$\begin{aligned} 3.3 & (42) & 2.6 & (18) & 3.8 & (14) \\ 9.1 & (114) & 7.3 & (51) & 7.4 & (27) \\ 87.6 & (1100) & 90.2 & (633) & 88.8 & (324)\end{aligned}$

$\begin{array}{rlccrl}8.1 & (102) & 10.3 & (72) & 12.3 & (45) \\ 13.2 & (166) & 15.2 & (107) & 15.3 & (56) \\ 60.2 & (755) & 59.1 & (415) & 58.4 & (213) \\ 16.9 & (212) & 13.5 & (95) & 13.4 & (49) \\ 1.6 & (20) & 1.9 & (13) & 0.5 & (2)\end{array}$

94.7 (1189)

95.4 (670)

$94.8 \quad(346)$

11.1 (139)

$17.3 \quad$ (121)

$20.5 \quad(75)$

$\begin{aligned} 4.7 & (58) \\ 38.2 & (476) \\ 11.6 & (145) \\ 45.5 & (568)\end{aligned}$

$5.7 \quad(40)$

38.9 (271)

12.9 (90)

42.5 (296)

$6.6 \quad(24)$

36.4 (133)

$14.8 \quad$ (54)

42.2 (154) $\begin{aligned} 2.4 & (30) \\ 32.5 & (408) \\ 2.9 & (36) \\ 62.2 & (781)\end{aligned}$

$1.4 \quad(10)$

$32.3 \quad$ (227)

$3.1 \quad(22.0)$

63.1 (443)

$2.5 \quad$ (9)

34.8 (127)

2.7 (10)

60.0 (219) 
Relative contribution to household income

Neither employed

$\begin{array}{llllll}7.6 & (95) & 6.5 & (33) & 6.8 & (19)\end{array}$

Partner contributes all / more

Respondent contributes all / more

$76.8 \quad(966)$

Both contribute the same

$5.6 \quad(70)$

$12.0 \quad 61)$

$76.4 \quad(214)$

$10.0 \quad(28$

$2.7 \quad(30)$

$1.6 \quad(10)$

$75.3 \quad(86)$

$76.1(469)$

$2.6 \quad(9)$

$6.9 \quad(35)$

$6.8 \quad(19)$

$11.9 \quad(131)$

$11.5 \quad(71.0)$

74.4 (258)

$11.5 \quad(40)$

$11.5 \quad(40)$ 


\begin{tabular}{|c|c|c|c|c|c|c|c|c|}
\hline & \multicolumn{4}{|c|}{ Dar Es Salaam (Age adjusted) } & \multicolumn{4}{|c|}{ Mbeya (Age adjusted) } \\
\hline & \multicolumn{2}{|c|}{ Lifetime IPV } & \multicolumn{2}{|c|}{ Current IPV } & \multicolumn{2}{|c|}{ Lifetime IPV } & \multicolumn{2}{|c|}{ Current IPV } \\
\hline & & $R \quad(95 \% \mathrm{Cl})$ & & OR $\quad(95 \% \mathrm{Cl})$ & & R $\quad(95 \% \mathrm{Cl})$ & & R $(95 \% \mathrm{Cl})$ \\
\hline Household crowding index & $1.10^{*}$ & $(1.10,1.21)$ & $1.15^{*}$ & $(1.03,1.27)$ & $1.13^{*}$ & $(1.01,1.26)$ & 1.10 & $(0.98,1.23)$ \\
\hline \multicolumn{9}{|l|}{ Household SES } \\
\hline High & 1.00 & & 1.00 & & 1.00 & & 1.00 & \\
\hline Medium & 1.10 & $(0.75,1.60)$ & 1.08 & $(0.71,1.63)$ & 1.17 & $(0.57,2.40)$ & 0.55 & $(0.25,1.21)$ \\
\hline Low & 1.25 & $(0.90,1.75)$ & 0.96 & $(0.60,1.54)$ & $1.97^{*}$ & $(1.05,3.70)$ & 0.74 & $(0.38,1.44)$ \\
\hline \multicolumn{9}{|l|}{ Respondent education } \\
\hline Some secondary or higher & 1.00 & & 1.00 & & 1.00 & & 1.00 & \\
\hline Completed primary & $1.49^{*}$ & $(1.14,1.94)$ & 1.31 & $(0.94,1.82)$ & 1.07 & $(0.70,1.64)$ & 1.08 & $(0.67,1.74)$ \\
\hline Some primary & $1.57^{*}$ & $(1.06,2.34)$ & $1.75^{*}$ & $(1.10,2.78)$ & $1.86^{*}$ & $(1.09,3.17)$ & 1.45 & $(0.81,2.60)$ \\
\hline None & 0.90 & $(0.62,1.31)$ & 0.77 & $(0.47,1.28)$ & $1.60^{*}$ & $(1.00,2.54)$ & 1.50 & $(0.89,2.53)$ \\
\hline \multicolumn{9}{|l|}{ Partner education } \\
\hline Some secondary or higher & 1.00 & & 1.00 & & 1.00 & & 1.00 & \\
\hline Completed primary & $1.54^{*}$ & $(1.23,1.95)$ & $1.36^{*}$ & $(1.03,1.80)$ & $1.51^{*}$ & $(1.11,2.06)$ & 1.30 & $(0.91,1.86)$ \\
\hline Some primary & 1.20 & $(0.77,1.89)$ & 1.15 & $(0.63,2.08)$ & $2.09 *$ & $(1.37,3.19)$ & $2.17^{*}$ & $(1.35,3.46)$ \\
\hline None & 1.07 & $(0.65,1.77)$ & 1.12 & $(0.60,2.10)$ & $2.76^{*}$ & $(1.65,4.59)$ & $3.45^{*}$ & $(2.04,5.84)$ \\
\hline \multicolumn{9}{|l|}{ Educational difference } \\
\hline Both have same & 1.00 & & 1.00 & & 1.00 & & 1.00 & \\
\hline Partner has more & 0.94 & $(0.75,1.19)$ & 0.96 & $(0.72,1.27)$ & 1.15 & $(0.90,1.48)$ & 1.16 & $(0.88,1.53)$ \\
\hline Respondent has more & 1.36 & $(0.96,1.92)$ & 1.30 & $(0.86,1.97)$ & $1.46^{*}$ & $(1.00,2.12)$ & $1.74 *$ & $(1.18,2.56)$ \\
\hline Both have no schooling & 0.40 & $(0.16,1.01)$ & 0.75 & $(0.25,2.26)$ & 1.77 & $(0.98,3.21)$ & $2.68^{*}$ & $(1.50,4.79)$ \\
\hline Respondent earns money & $1.30^{*}$ & $(1.05,1.61)$ & 1.20 & $(0.92,1.56)$ & 1.05 & $(0.83,1.33)$ & 0.95 & $(0.74,1.24)$ \\
\hline Partner working & 1.21 & $(0.88,1.67)$ & 1.05 & $(0.71,1.56)$ & 1.46 & $(0.88,2.42)$ & 0.96 & $(0.55,1.68)$ \\
\hline \multicolumn{9}{|l|}{ Relative employment } \\
\hline Both working & 1.00 & & 1.00 & & 1.00 & & 1.00 & \\
\hline Partner only working & $0.77^{*}$ & $(0.61,0.97)$ & 0.83 & $(0.63,1.09)$ & 1.01 & $(0.79,1.29)$ & 1.06 & $(0.81,1.38)$ \\
\hline Respondent working & 0.82 & $(0.52,1.29)$ & 0.94 & $(0.53,1.67)$ & 1.04 & $(0.52,2.08)$ & 1.21 & $(0.56,2.59)$ \\
\hline Neither working & 0.66 & $(0.42,1.03)$ & 0.81 & $(0.47,1.40)$ & 0.42 & $(0.19,0.91)$ & 0.92 & $(0.41,2.07)$ \\
\hline \multicolumn{9}{|l|}{ Contribution to household income } \\
\hline Both contribute the same & 1.00 & & 1.00 & & 1.00 & & 1.00 & \\
\hline Partner contributes all / more & 0.65 & $(0.40,1.06)$ & $0.55^{*}$ & $(0.31,0.97)$ & 1.33 & $(0.92,1.93)$ & 1.44 & $(0.84,2.49)$ \\
\hline Respondent contributes all / more & 0.91 & $(0.51,1.63)$ & 0.75 & $(0.38,1.48)$ & 1.68 & $(0.99,2.83)$ & 0.99 & $(0.66,1.48)$ \\
\hline Neither working & 0.54 & $(0.40,1.06)$ & 0.51 & $(0.24,1.08)$ & 0.54 & $(0.23,1.24)$ & 0.84 & $(0.35,2.01)$ \\
\hline
\end{tabular}

$* p<0.05$ 
Table 4: Respondent attitudes to wife beating

\begin{tabular}{|c|c|c|c|c|}
\hline & \multicolumn{2}{|c|}{ Dar Es Salaam } & \multicolumn{2}{|c|}{ Mbeya } \\
\hline & $\begin{array}{l}\text { At least one good } \\
\text { reason to hit }\end{array}$ & Can refuse sex & $\begin{array}{l}\text { At least one good } \\
\text { reason to hit }\end{array}$ & Can refuse sex \\
\hline \multicolumn{5}{|l|}{ Education attainment } \\
\hline Some secondary or more & $36.0 \quad(117)$ & $97.6 \quad(320)$ & 49.0 (48) & $99.0(97)$ \\
\hline Complete primary & 71.4 (539) & 95.3 (733) & 69.8 (494) & 85.9 (609) \\
\hline Some primary & 78.9 (116) & $94.6(140)$ & $80.0 \quad(108)$ & 82.7 (110) \\
\hline None & 76.2 (144) & 92.1 (174) & $72.6 \quad(215)$ & $86.3 \quad(258)$ \\
\hline Chi-square (test for trend) & $<0.001$ & 0.005 & 0.001 & 0.081 \\
\hline Doesn't earn money & $68.8(499)$ & $94.4(693)$ & 75.8 & $86.0(375)$ \\
\hline Earns money & $60.3(417)$ & $96.3(674)$ & $66.8(540)$ & 87.0 (699) \\
\hline Chi-square & 0.001 & 0.093 & 0.001 & 0.607 \\
\hline \multicolumn{5}{|l|}{ Household SES } \\
\hline High & $37.1(62)$ & 96.5 (166) & 46.3 (19) & $95.2(40)$ \\
\hline Medium & $52.1(173)$ & $97.0 \quad(327)$ & $62.3(71)$ & $91.2(103)$ \\
\hline Low & $74.3(681)$ & $94.5(874)$ & 89.6 (775) & 85.9 (931) \\
\hline Chi-square (test for trend) & $<0.001$ & 0.079 & 0.001 & 0.074 \\
\hline
\end{tabular}

